\title{
Antidiabetic medication and risk of dementia in patients with type 2 diabetes: a nested case-control study
}

\author{
Ida Kim Wium-Andersen ${ }^{1}$, Merete Osler ${ }^{2,3}$, Martin Balslev Jørgensen ${ }^{1}$, Jørgen Rungby ${ }^{4}$ and \\ Marie Kim Wium-Andersen ${ }^{2}$ \\ ${ }^{1}$ Psychiatric Center Copenhagen, Copenhagen $\varnothing$, Denmark, ${ }^{2}$ Center for Clinical Research and Prevention, \\ Frederiksberg Hospital, Frederiksberg, Denmark, ${ }^{3}$ Section of Epidemiology, Department of Public Health, University \\ of Copenhagen, Copenhagen K, Denmark, and ${ }^{4}$ Department of Endocrinology and Copenhagen Center for \\ Translational Research, Bispebjerg Hospital, Copenhagen NV, Denmark \\ Correspondence \\ should be addressed \\ to I K Wium-Andersen \\ Email \\ Ida.kim.wiumandersen@ \\ regionh.dk
}

\begin{abstract}
Objective: Diabetes is a risk factor for dementia, but whether antidiabetic medication decreases the risk is unclear. We examined the association between antidiabetic medication and dementia.

Design: We performed a nested case-control study within a cohort of all 176250 patients registered with type 2 diabetes in the Danish National Diabetes Register between 1995 and 2012. This population was followed for dementia diagnosis or anti-dementia medication use until May 2018. Using risk-set sampling, each dementia case $(n=11619)$ was matched on follow-up time and calender year of dementia with four controls randomly selected among cohort members without dementia ( $n=46476$ ). Ever use and mean daily defined dose of antidiabetic medication was categorized in types (insulin, metformin, sulfonylurea and glinides combined, glitazone, dipeptidyl peptidase 4 (DPP4) inhibitors, glucagonlike peptide 1 (GLP1) analogs, sodium-glucose transport protein 2 (SGLT2) inhibitors and acarbose).

Methods: Conditional logistic regression models were fitted to calculate odds ratios (ORs) for dementia associated with antidiabetic medication use, adjusting for potential confounders.

Results: Use of metformin, DPP4 inhibitors, GLP1 analogs, and SGLT2 inhibitors were associated with lower odds of dementia after multible adjustments (ORs of 0.94 (95\% confidence interval (CI): 0.89-0.99), 0.80 (95\% CI 0.74-0.88), 0.58 (95\% Cl: 0.50-0.67), and 0.58 (95\% Cl: 0.42-0.81), respectively), with a gradual decrease in odds of dementia for each increase in daily defined dose. Analyses of the most frequent treatment regimes did not show any synergistic effects of combined treatment.

Conclusion: Use of metformin, DPP4 inhibitors, GLP1 analogs and SGLT2 inhibitors was associated with lower risk of dementia in patients with diabetes.
\end{abstract}

\section{Introduction}

Dementia is a disease characterized by progressive irreversible cognitive damage. In 2015, 47 million people were living with dementia, and numbers are predicted to triple by 2050 (1). Diabetes mellitus is a well-established risk factor for dementia, and particularly patients with type 2 diabetes seem to have increased risk of dementia
(2). This risk may be caused by a shared pathophysiology of diabetes and dementia involving hyperglycemia and hyperinsulinemia (3), which raises the question of whether use of antidiabetic medications can reduce risk of dementia in patients with diabetes. Only few studies have examined this with equivocal results and, although

Published by Bioscientifica Ltd. 
combination of treatment is common, none have studied combination therapy or included the newest antidiabetic medications such as dipeptidyl peptidase 4 (DPP4) inhibitors, glucagon-like peptide 1 (GLP1) analogs, and sodium-glucose transport protein 2 (SGLT2) inhibitors. Of those, especially GLP1 analogs are of interest as they have been shown to improve cognition in rodents $(4,5,6)$ and to improve cerebral metabolism in Alzheimer's disease (7).

As one in eleven (425 millions) adults worldwide suffer from diabetes (8), it is important to know whether the higher risk of developing dementia in patients with diabetes could be reversed by antidiabetic medication. Moreover, it is important to establish if any effect is related to specific types of antidiabetic medication or combinations of treatment.

Against this background, we investigated whether different types of antidiabetic medication and treatment regimens (combinations of antidiabetic drugs) were associated with risk of developing dementia in Danish patients with type 2 diabetes.

\section{Patients and methods}

\section{Study population and follow-up}

The study was based on patients in Denmark registered with type 2 diabetes in the National Diabetes Register (NDR) between January 1, 1995 and December 31, 2012 $(n=178$ 403). Inclusion in this register is based on a validated algorithm combining information on diabetes ascertained by physician's diagnosis (International Classification of Disease 10 (ICD10) codes DE10-14, DH36.0, DO24) in the Danish National Patients Registry (NPR) after 1990, prescriptions of antidiabetic medication in the Danish Prescription Register, and measures of blood glucose or referrals to feet therapists from the National Health Insurance Service Register (9). The data from the NDR were compared with historical data from the NPR and Danish Adult Diabetes Registry to improve validity (10). Patients with diabetes due to gestational diabetes (DO24.4-9; $n=1806)$, starvation (DE12; $n=347$ ), patients with a diagnosis of type 2 diabetes under the age of $35(n=3695)$, or a diagnosis of dementia or use of anti-dementia medication before registration in the NDR $(n=2138)$ were excluded (Fig. 1). Dementia diagnoses and dementia medication were tracked back to the establishment of the NPR and Danish National Prescription Registry (DPR) in 1977 and 1995, respectively. That left a study population of 170417 patients followed until first dementia diagnosis or prescription of dementia medication, emigration or death in the NPR and the Danish Civil Registration System or until May 2018. During a median follow-up of 7.2 years (range: 0.0-22.8 years, IQR: 3.5-11.5 years), we identified 11619 cases of incident dementia (only diagnosis: $n=7473$; diagnosis and medication: $n=3259$; only medication: $n=887$ ). A casecontrol study was nested within the cohort of patients with type 2 diabetes using risk-set sampling generated by the procedure sttocc in Stata. Thus, we randomly selected controls among members in the cohort who remained dementia free and matched them on follow-up time and calendar year to case patients in a 1:4 ratio $(n=46476)$. The matching on calendar year was to ensure that both cases and controls were followed in the same calendar time ensuring equal access to for example the newer antidiabetic drugs. The rationale for choosing a nested case-control study instead of a prospective cohort design was that this design allowed us to calculate the amount of antidiabetic medication used in the study period while avoiding immortal time bias.

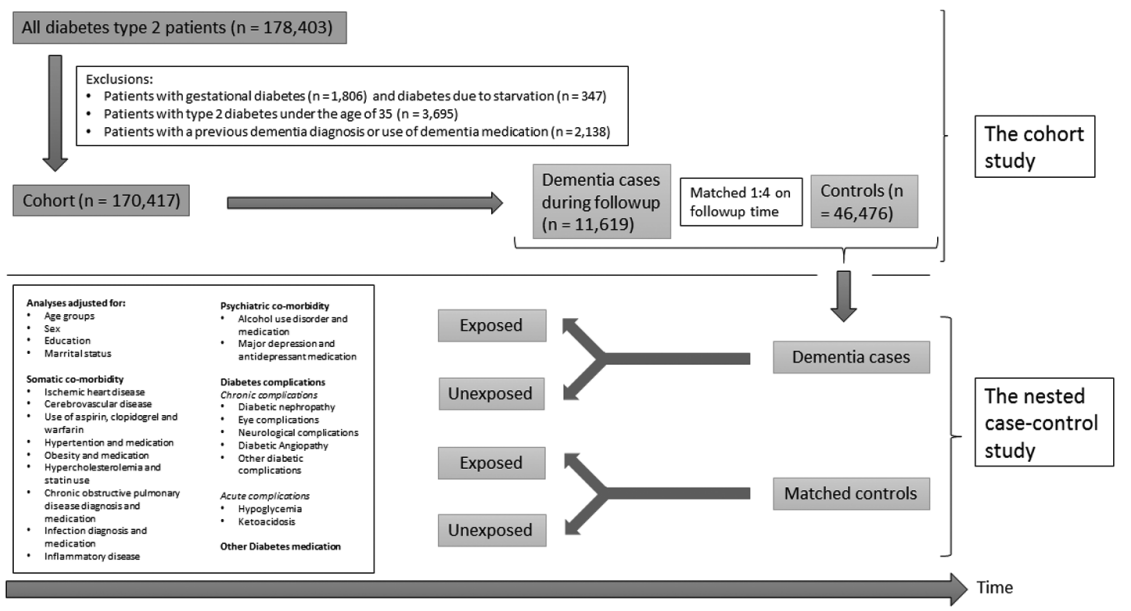

Figure 1

Flowchart of the study design. 


\section{Outcome: dementia}

Dementia was defined as an International Classification of Diseases 10th revision (ICD10) diagnosis of F00-F04 and G30, registered in the NPR which holds data on diagnosis and dates from all somatic and psychiatric hospital contacts for both in- and outpatients in Denmark (11). From the DPR, information on prescriptions of antidementia medication was obtained using the anatomic therapeutic codes (ATCs) N06D.

\section{Exposure: antidiabetic medication}

We obtained all prescriptions of antidiabetic medication from January 1995 to the index date for both cases and controls from the DPR using ATC codes A10. For each prescription, the DPR records date and a full description of the dispensed product, including ACT and the total number of daily defined doses (DDD). The DDD was define by the World Health Organization as 'the assumed average maintenance dose per day for a drug used for its main indication in adults' (12) and is thus in our study used as a measurement of dosage per time. We categorized antidiabetic medication into the following types: insulin, metformin, sulfonylureas and glinides combined, glitazones, DPP4 inhibitors, GLP1 analogs, SGLT2 inhibitors and acarbose (Supplementary Table 1, see section on supplementary data given at the end of this article). Mean DDD was calculated for each type of antidiabetic medication and classified into sufficiently sized groups and consequently cut points differed across types of medication. As many patients used more than one type of antidiabetic medication, we also evaluated any added effect of combined treatment. We first generated all treatment regimens (a total of 128 different combinations of the antidiabetic medication types mentioned above) used in our diabetes population and extracted the 12 most commonly used (which included $>1000$ patients) while all other combinations used formed one single category termed 'other combinations' (Supplementary Table 2). However, as none of the most common combinations included SGLT2 inhibitors, we further categorized 'other combinations' into 'other combinations including SGLT2' and 'other combinations not including SGLT2'.

\section{Covariables}

Age was included as a continuous variable. Education was categorized as basic (7-9 grade of obligatory schooling), medium (high school degree/vocational), higher education (more than high school degree), or unknown based on data from the Integrated Database for Labor Market Research. Information on marital status was obtained from the Danish Civil Registration System and categorized as married, unmarried, divorced or widowed. From the NPR and the DPR, we included information on hospitalization and medication for somatic or psychiatric diseases five years preceding study entry (i.e. diabetes diagnosis). Comorbidity and co-medication for ischemic heart disease, cerebrovascular disease, hypertension, obesity, hypercholesterolemia, infections, chronic obstructive pulmonary disease, inflammatory disorder, depression and alcohol use disorder was included. Finally, we included information on acute and chronic diabetes complications and the number of hospitalizations with complications after diabetes diagnosis. Acute complications included occasions with ketoacidosis or hypoglycemia, while chronic complications included diabetic nephropathy, eye complications, neurological complications, or diabetic angiopathy (Supplementary Table 1). The International Classification of Diseases 10th revision (ICD10) codes and the ATCs are shown in Supplementary Table 1 . Finally, in a subgroup of patients from the Danish Adult Diabetes Registry (DADR), we included information on self-reported smoke status (never, previous or current smoking), BMI, hemoglobin A1c $(\mathrm{mmol} / \mathrm{mol})$, low-density lipoprotein cholesterol $(\mathrm{mmol} / \mathrm{L})$, and systolic blood pressure. The DADR is a database initiated in 1999, which includes clinical information on diabetes patients, and since 2004 it has been mandatory to report data to the register from outpatients clinics and hospitals (13).

\section{Data approval}

The study was approved by the Danish Data Inspection. All data were retrieved from administrative registers, and informed consent was not required of participants. Data in this study are anonymized data located at Statistics Denmark and at the Danish Clinical Registries.

\section{Statistical analysis}

Stata version 15 (StataCorp, College Station, TX, USA) was used for statistical analyses. Conditional logistic regression was used to calculate ORs using four levels of adjustment: (1) unadjusted, (2) adjusted for age groups, sex, education, (3) adding marital status, comorbidity, co-medication and number of acute and chronic diabetes complications, and finally (4) adding mutually adjustment for other types of antidiabetic medications. 
We repeated all analyses with adjustment for diabetes complication type (e.g. hypoglycemia, ketoacidosis, retinopathy, nephropathy etc.) instead of number of complications, but results were similar (data not shown).

In a supplementary analysis, we identified cases and controls with data in the DADR $(n=24$ 182). Patients with a registered date for examination and blood tests in the DADR after the dementia date or corresponding date for controls ( $n=11236)$ were excluded. After these exclusions any patient lacking a corresponding control was also excluded and vice versa. Left with 5449 patients in this subgroup, we repeated analyses with further inclusion of the clinical variables from the DADR.

\section{Results}

Our study population consisted of 11619 dementia cases and 46476 controls. Cases were more often women, had higher mean age, were less educated and showed higher prevalence of most comorbidities and used all antidiabetic medication types less often except sulfonylureas and acarbose (Table 1). Overall, 9122 (79\%) of cases and 38 $572(83 \%)$ of controls had used antidiabetic medication (unadjusted OR: 0.75 (95\% confidence interval (CI): 0.710.78), multiple adjusted OR: 0.93 (95\% CI: 0.87-0.99)). Characteristics of users of antidiabetic drugs versus nonusers are shown in Supplementary Tables 3, 4, 5, 6, 7, 8, 9, 10 and 11.

Use of any type of antidiabetic medication except for sulfonylureas and acarbose were associated with lower odds of dementia in the unadjusted models (Fig. 2). After multiple adjustments, including use of other antidiabetic medications, use of insulin, metformin, DPP4 inhibitors, GLP1 analogs, and SGLT2 inhibitors remained associated with lower odds of dementia with the strongest estimates for GLP1 analogs and SGLT2 inhibitors (ORs of 0.58 (95\% CI: 0.50-0.67) and 0.58 (95\% CI: 0.42-0.81) respectively). Sulfonylureas were associated with slightly higher odds of dementia (OR: 1.07 (95\% CI: 1.02-1.13)).

Further, increasing DDD of different types of antidiabetic medications, metformin, DPP4 inhibitors, and GLP1 analogs showed a gradual decrease in odds of dementia (Fig. 3). Insulin and sulfonylureas were not associated with dementia in a dose-dependent manner.

In the analyses of the most frequent treatment regimes, combinations with DPP4 inhibitors, GLP1 analogs, and SGLT2 inhibitors were associated with lower odds of dementia after adjustments with the lowest odds of the combinations including SGLT2 inhibitors
Table 1 Main demographic and clinical characteristics of the included patients with type 2 diabetes from the National Diabetes Register.

\begin{tabular}{|c|c|c|}
\hline & Dementia cases & Controls \\
\hline Number & 11619 & 46476 \\
\hline Age (years), mean (IQR) & $70.8(51-67)$ & $59.0(64-78)$ \\
\hline Men, $n(\%)$ & $5566(48)$ & $25900(56)$ \\
\hline \multicolumn{3}{|l|}{ Education, $n(\%)$} \\
\hline Short education & $5522(48)$ & $20112(43)$ \\
\hline Medium education & $3187(27)$ & $17566(38)$ \\
\hline Long education & $918(8)$ & $5105(11)$ \\
\hline Unknown & $1992(17)$ & $3693(8)$ \\
\hline \multicolumn{3}{|l|}{ Marrital status, $n(\%)$} \\
\hline Married & $6027(52)$ & $28720(62)$ \\
\hline Unmarried & $820(7)$ & $5696(12)$ \\
\hline Divorced & $1538(13)$ & $6682(14)$ \\
\hline Widow/widower & $3176(27)$ & $5106(11)$ \\
\hline Unknown & $58(0.5)$ & $272(1)$ \\
\hline $\begin{array}{l}\text { Ischemic heart disease, } \\
n(\%)\end{array}$ & $1502(13)$ & $4589(10)$ \\
\hline $\begin{array}{l}\text { Cerebrovascular disease, } \\
n(\%)\end{array}$ & $1011(9)$ & $1881(4)$ \\
\hline $\begin{array}{l}\text { Clopidogrel, warfarin, } \\
\text { aspirin use, } n(\%)\end{array}$ & $4232(36)$ & $10655(23)$ \\
\hline Hypertension, $n(\%)$ & $7229(62)$ & $24325(52)$ \\
\hline Obesity, $n(\%)$ & $1054(9)$ & $7231(16)$ \\
\hline $\begin{array}{l}\text { Hypercholesterolemia, } \\
n(\%)\end{array}$ & $2190(19)$ & 9044 (19) \\
\hline Infections, $n(\%)$ & $2363(20)$ & $7322(16)$ \\
\hline $\begin{array}{l}\text { Chronic obstructive } \\
\quad \text { pulmonary disease, } n(\%)\end{array}$ & $1718(15)$ & $6060(13)$ \\
\hline $\begin{array}{l}\text { Inflammatory disorder, } \\
n(\%)\end{array}$ & $543(5)$ & $1880(4)$ \\
\hline Depression, $n(\%)$ & $2458(21)$ & $6980(15)$ \\
\hline $\begin{array}{l}\text { Alcohol dependence, } \\
n(\%)\end{array}$ & $536(5)$ & $1655(4)$ \\
\hline \multicolumn{3}{|c|}{ Chronic diabetes complications, $n(\%)$} \\
\hline 0 complications & $6579(57)$ & $26793(58)$ \\
\hline 1 complication & $2880(25)$ & $11195(24)$ \\
\hline 2 complications & $1236(11)$ & $4894(11)$ \\
\hline 3 complications & $562(5)$ & $2082(4)$ \\
\hline$\geq 4$ complications & $362(3)$ & $1512(3)$ \\
\hline \multicolumn{3}{|c|}{ Acute diabetes complications, $n(\%)$} \\
\hline 0 complications & $11243(97)$ & $45268(97)$ \\
\hline$\geq 1$ complications & $376(3)$ & $1208(3)$ \\
\hline $\begin{array}{l}\text { Antidiabetic medication } \\
\text { use, no. of ever- } \\
\text { users (\%) }\end{array}$ & $9122(79)$ & $35078(82)$ \\
\hline \multicolumn{3}{|l|}{ Any type } \\
\hline Insulin & $2425(21)$ & $12267(26)$ \\
\hline Metformin & $6279(54)$ & $30894(66)$ \\
\hline Sulfonylureas & $6704(58)$ & $26276(57)$ \\
\hline Glitazones & $271(2)$ & $2015(4)$ \\
\hline DPP4 inhibitors & $902(8)$ & $6121(13)$ \\
\hline GLP1 analogs & $246(2)$ & $3922(8)$ \\
\hline SGLT2 inhibitors & $44(0.4)$ & $868(2)$ \\
\hline Acarbose & $197(2)$ & $833(2)$ \\
\hline
\end{tabular}

Based on 11619 cases and 46476 controls.

DDD, daily defined dose; DPP4, dipeptidyl peptidase 4; GLP1, glucagonlike peptide 1; SGLT2, sodium-glucose transport protein 2. 
(OR of 0.39 (95\% CI: 0.29-0.55)) (Fig. 4). However, the observed odds due to more than one type of treatment were nearly identical to the expected odds from the multiplicative models for the respective types of medications provided in Fig. 2.

In the supplementary analysis of the subgroup of patients included in the DADR, a total of 1446 cases and 4003 controls had clinical data available. Demographic and clinical characteristics of this subgroup are shown in Supplementary Table 12. After multiple adjustments including other antidiabetic medications, only GLP1 analogs and SGLT2 inhibitors were associated with significantly lower ORs of 0.59 (95\% CI: 0.45-0.77) and 0.43 (95\% CI: 0.22-0.83) (Supplementary Fig. 1). Further adjustment for clinical variables including HbA1c did not change the ORs. In analyses of DDD, both groups of GLP1 analogs stayed significant after adjustment for clinical variables (Supplementary Fig. 2).

\section{Discussion}

In this nationwide nested case-control study, we demonstrated that patients with diabetes who used metformin, DPP4 inhibitors, GLP1 analogs, or SGLT2 inhibitors had lower odds of developing dementia even after adjustment for potential confounders and for use of other types of antidiabetic medication. The association seemed dependent on dose and duration of treatment. In the combined analysis, combinations with GLP1 analogs, DPP4 inhibitors, and SGLT2 inhibitors were associated with the lowest odds of dementia. The odds were nearly identical to the expected odds from a multiplicative model for the respective types of medications suggesting that the effect of combined use depends only on the individual drugs in the combination with no synergistic effect. Insulin seemed associated with lower risk of diabetes in the analysis of ever use of insulin (Fig. 2); however, this could not be replicated in the later analyses.

The results of our study, suggesting that use of specific types of antidiabetic medication are associated with lower risk of dementia, are supported by other studies, but direct comparison is difficult since previous studies included older antidiabetic medications only. Two studies based on National Health Insurance Research Database from Taiwan showed that in patients with diabetes, use of sulfonylureas and metformin were associated with a decreased risk of dementia compared to patients not using antidiabetic

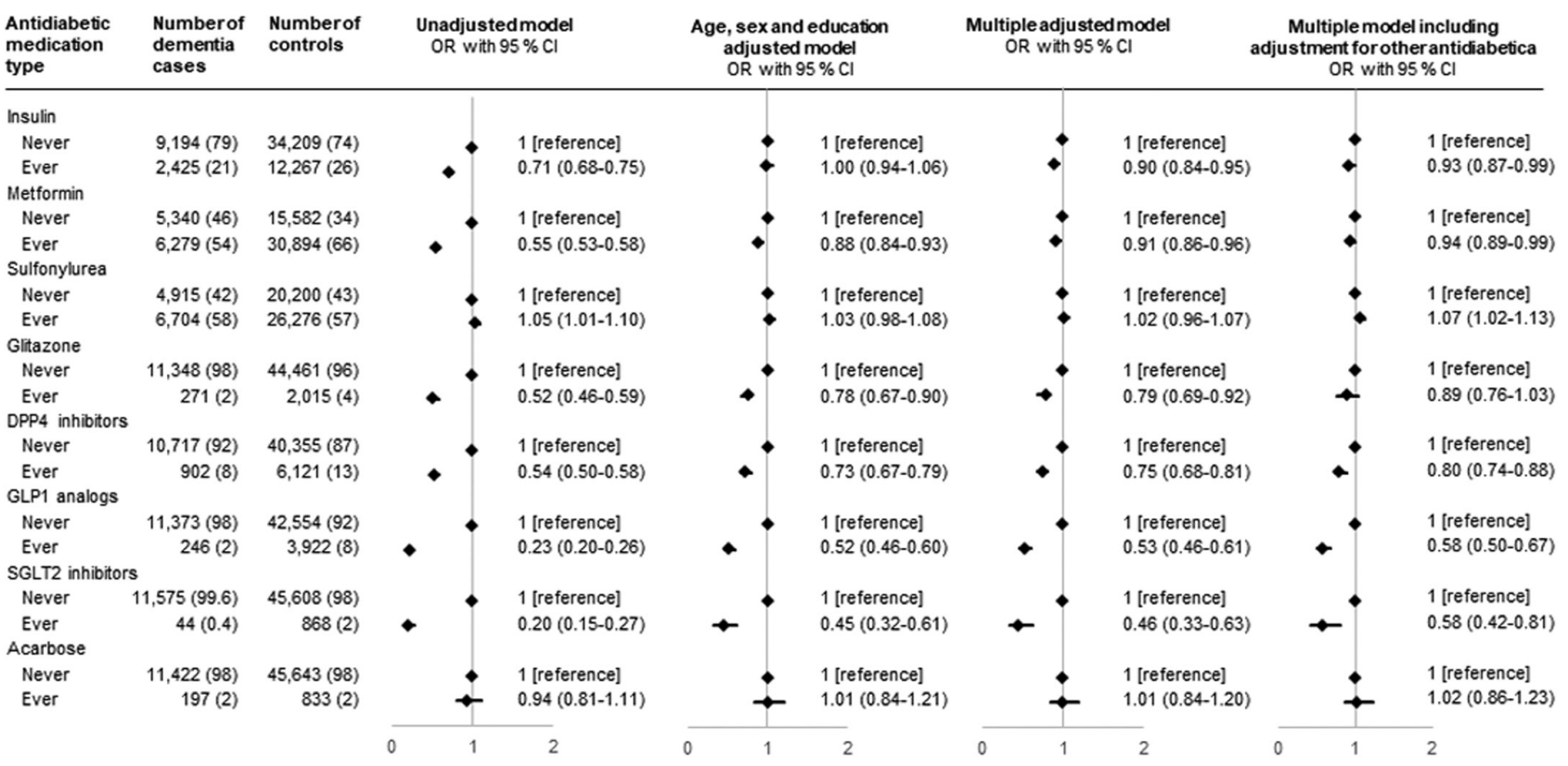

Figure 2

Use of antidiabetic medication in type 2 diabetic patients with (cases) or without (controls) dementia during follow-up in the National Diabetes Register, based on 11,619 cases and 46,476 controls. Multiple adjustments include age, sex, education, marital status, year of dementia diagnosis, ischemic heart disease, cerebrovascular disease, clopidogrel/warfarin/aspirin use, hypertension, obesity, hypercholesterolemia, infections, chronic obstructive pulmonary disease, inflammatory disorder, depression, alcohol use disorder, and number of acute and chronic diabetes complications. 


\begin{tabular}{|c|c|c|c|c|c|c|c|}
\hline $\begin{array}{l}\text { Mean DDD } \\
\text { during } \\
\text { follow-up }\end{array}$ & $\begin{array}{l}\text { Numberin } \\
\text { the case } \\
\text { group }\end{array}$ & $\begin{array}{l}\text { Numberin } \\
\text { the control } \\
\text { group }\end{array}$ & $\begin{array}{l}\text { Unadjustedmodel } \\
\text { OR with } 95 \% \mathrm{Cl}\end{array}$ & $\begin{array}{l}\text { Age, sex and education } \\
\text { adjusted model } \\
\text { OR with } 95 \% \mathrm{Cl}\end{array}$ & $\begin{array}{c}\text { Multifactorially adjusted } \\
\text { model } \\
\text { OR with } 95 \% \mathrm{Cl}\end{array}$ & $\begin{array}{r}\text { Multifactorial mod } \\
\text { adjustment forothe } \\
\text { OR with } 95\end{array}$ & $\begin{array}{l}\text { lel including } \\
\text { rantidiabetica } \\
\% \mathrm{Cl}\end{array}$ \\
\hline \multicolumn{8}{|l|}{ Insulin } \\
\hline$>0-1 \mathrm{DDD}$ & $2,120(18)$ & $10,220(22)$ & $0.75(0.71-0.79)$ & $1.00(0.94-1.06)$ & $0.90(0.84-0.96)$ & & $0.93(0.87-0.99)$ \\
\hline $1-2 \mathrm{DDD}$ & $263(2)$ & $1,757(4)$ & $0.54(0.47-0.62)$ & $1.02(0.88-1.18)$ & $0.87(0.75-1.02)$ & & $0.91(0.78-1.06)$ \\
\hline$>2 \mathrm{DDD}$ & $42(0.4)$ & $290(0.6)$ & $0.52(0.37-0.72)$ & $1.20(0.84-1.71)$ & $0.99(0.69-1.43)$ & & $1.10(0.76-1.60)$ \\
\hline \multicolumn{8}{|l|}{ Metformin } \\
\hline$>0-0.5 \mathrm{DDD}$ & $4,352(37)$ & $17,960(39)$ & $0.66(0.63-0.69)$ & $0.94(0.89-0.99)$ & $0.96(0.91-1.02)$ & & $0.97(0.92-1.03)$ \\
\hline$>0.5-0.75 \mathrm{DDD}$ & $1,115(10)$ & $6,743(15)$ & $0.45(0.41-0.48)$ & $0.77(0.71-0.84)$ & $0.79(0.73-0.86)$ & & $0.85(0.78-0.93)$ \\
\hline$>0.75-1 \mathrm{DDD}$ & $611(5)$ & $4,439(10)$ & $0.38(0.34-0.41)$ & $0.80(0.72-0.88)$ & $0.83(0.75-0.92)$ & & $0.93(0.83-1.03)$ \\
\hline$>1 \mathrm{DDD}$ & $201(2)$ & $1,752(4)$ & $0.32(0.28-0.37)$ & $0.70(0.59-0.82)$ & $0.74(0.62-0.87)$ & & $0.81(0.68-0.95)$ \\
\hline \multicolumn{8}{|l|}{ Sulfonylurea } \\
\hline$O D D D$ & $4,915(42)$ & $20,200(43)$ & 1 [reference] & 1 [reference] & 1 [reference] & & 1 [reference] \\
\hline$>0-0.5 \mathrm{DDD}$ & $3,322(29)$ & $13,405(29)$ & $1.02(0.97-1.08)$ & $1.06(1.00-1.13)$ & $1.03(0.97-1.10)$ & & $1.09(1.02-1.16)$ \\
\hline$>0.5-0.75 \mathrm{DDD}$ & $1,111(10)$ & $4,139(9)$ & $1.11(1.02-1.19)$ & $1.04(0.96-1.14)$ & $1.04(0.95-1.13)$ & & $1.10(1.01-1.20)$ \\
\hline$>0.75-1 \mathrm{DDD}$ & $801(7)$ & $2,961(6)$ & $1.12(1.03-1.22)$ & $0.99(0.90-1.10)$ & $0.99(0.89-1.09)$ & & $1.06(0.96-1.17)$ \\
\hline \multicolumn{8}{|c|}{ DPP4 inhibitors } \\
\hline ODDD & $10,717(92)$ & $40,355(87)$ & 1 [reference] & 1 [reference] & 1 [reference] & & 1 [reference] \\
\hline$>0-0.1 \mathrm{DDD}$ & $390(3)$ & $2,530(5)$ & $0.56(0.50-0.63)$ & $0.77(0.68-0.87)$ & $0.79(0.70-0.90)$ & 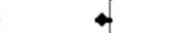 & $0.86(0.76-0.98)$ \\
\hline$>0.1 \mathrm{DDD}$ & $512(4)$ & $3,591(8)$ & $0.53(0.48-0.58)$ & $0.70(0.63-0.78)$ & $0.71(0.64-0.80)$ & $\bullet$ & $0.78(0.70-0.88)$ \\
\hline \multicolumn{8}{|l|}{ GLP1 analogs } \\
\hline ODDD & $11,373(98)$ & $42,554(92)$ & 1 [reference] & 1 [reference] & 1 [reference] & ] & 1 [reference] \\
\hline$>0-0.2 \mathrm{DDD}$ & $159(1)$ & $2,168(5)$ & $0.26(0.22-0.31)$ & $0.59(0.49-0.70)$ & $0.60(0.50-0.72)$ & 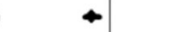 & $0.67(0.56-0.80)$ \\
\hline \multirow[t]{2}{*}{$>0.2 \mathrm{DDD}$} & $87(1)$ & $1,754(4)$ & $0.18(0.14-0.22)$ & $0.44(0.35-0.55)$ & $0.43(0.34-0.54)$ & 4 & $0.49(0.38-0.62)$ \\
\hline & & & 1 & 2 & 0 & 1 & \\
\hline
\end{tabular}

\section{Figure 3}

Previous use of antidiabetic medication in type 2 diabetic patients with (cases) or without (controls) dementia during follow-up in the National Diabetes Register based on 11,619 cases and 46,476 controls. Multifactorial is adjusted for age, sex, education, marital status, year of dementia diagnosis, ischemic heart disease, cerebrovascular disease, clopidogrel/warfarin/aspirin use, hypertension, obesity, hypercholesterolemia, infections, chronic obstructive pulmonary disease, inflammatory disorder, depression, alcohol use disorder, and number of acute and chronic diabetes complications.

medications (14), while metformin users seemed to have lower rates of dementia compared to sulfonylurea and glitazone users (15). Similarly, Orkaby et al. recently found that metformin decreased the risk of dementia when compared to sulfonylureas in US veterans younger than 75 years (16). This is in line with our finding that metformin use was associated with lower odds of dementia. Metformin may reduce dementia risk by reducing oxidative stress, inflammation, and apoptosis and by suppressing the damaging effects of advanced glycosylation end products which are produced during hyperglycemia and have potential toxic effects on neurons $(17,18)$.

By contrast, a case-control study including 7086 cases with Alzheimer's dementia (AD) recruited among patients from general practice in the United Kingdom found that long-term use of metformin was associated with increased odds of $\mathrm{AD}$, while insulin, sulfonylureas, and glitazones were not as compared to non-users without diabetes (19). In our study, we did not find any consistent associations of insulin, sulfonylureas, or glitazones with dementia even though previous randomized controlled studies have suggested that especially insulin and glitazones have pro-cognitive effects (20). It may be that the antidiabetic medications which benefit dementia patients without diabetes are not the same drugs that may prevent dementia in diabetes patients.

No population-based study so far has examined the influence of newer antidiabetic medications on dementia risk. However, many studies have described GLP1's possible protective role on cognitive symptoms $(7,21)$ and risk of dementia and several rodent studies have suggested that GLP-1 analogs improve cognition $(5,6)$. Mechanistically, GLP1 may cross the blood-brain barrier and exhibit procognitive effects by improving neurogenesis, protect against apoptosis, inflammation and oxidative stress and prevent amyloid plaque formation in the brain (22) and improve cerebral glucose metabolism $(7,23)$.

DPP4 inhibitors inhibit the enzyme responsible for degradation of GLP1 hereby doubling the concentration of active GLP1 (24). Compared to GLP1 analogs, DPP4 inhibitors increase active GLP1 levels considerably less resulting in lower impact on both glycemic and extraglycemic effects (e.g. body weight and blood pressure) (25). This is in accordance with the results seen in our study, where the associations for DPP4 inhibitor use were weaker than for GLP1 analog use but with the same tendency. 


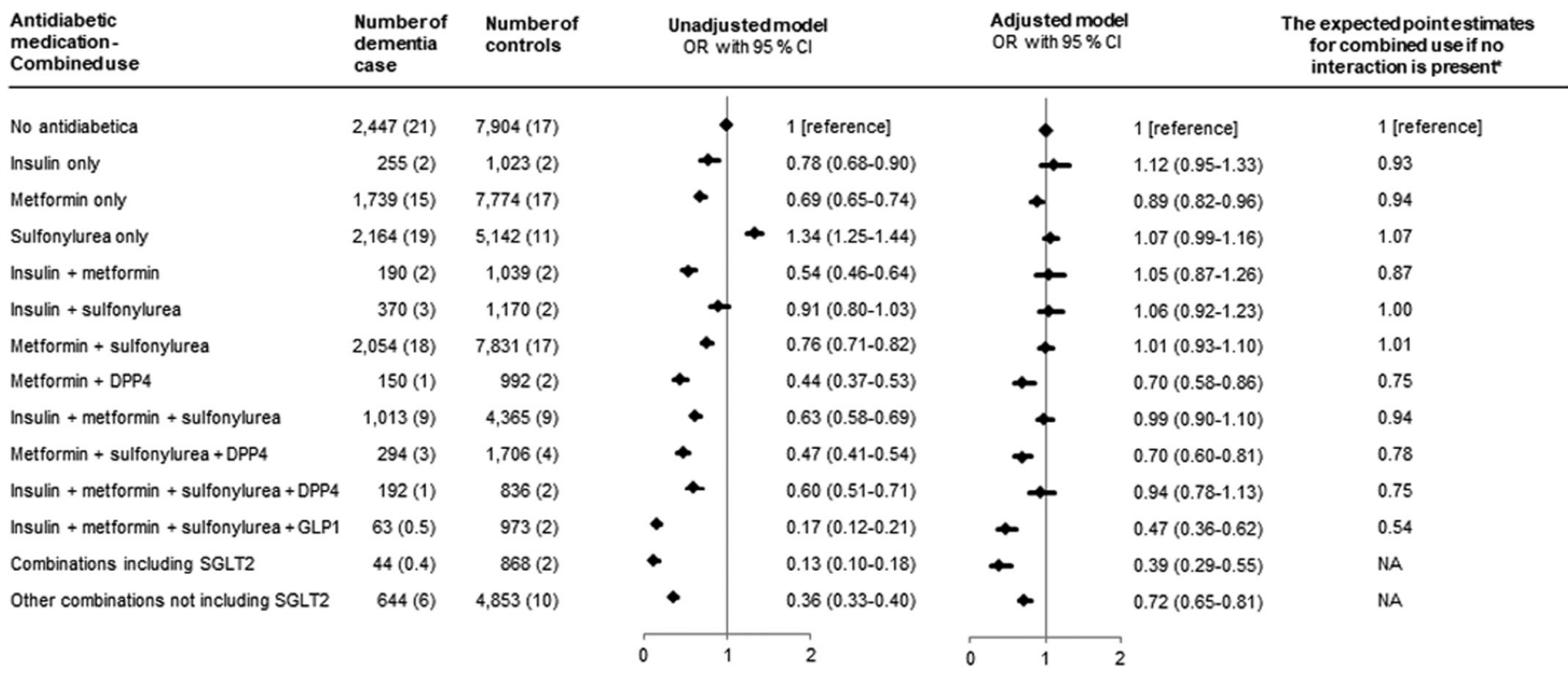

\section{Figure 4}

Combinations of antidiabetic medication in type 2 diabetic patients with (cases) or without (controls) dementia during follow-up in the National Diabetes Register. Based on 11,619 cases and 46,476 controls. Adjustment for age, sex, education, marital status, year of dementia diagnosis, ischemic heart disease, cerebrovascular disease, clopidogrel/warfarin/aspirin use, hypertension, obesity, hypercholesterolemia, infections, chronic obstructive pulmonary disease, inflammatory disorder, depression, alcohol use disorder, and number of acute and chronic diabetes complications. *The expected point estimates for combined use was calculated using the risk estimates in Figure 1.

SGLT2 inhibitors are a new drug class which reduces plasma glucose by increased renal excretion (26). Two rodent studies have indicated that SGLT2 inhibitors may prevent cognitive decline due to reduced oxidative stress and inflammation, improved brain mitochondrial function and plasticity in the hippocampus which is particularly affected by type 2 diabetes $(27,28)$. Furthermore, it has been suggested that SGLT2 inhibitors may inhibit the enzyme acetylcholinesterase, the primary target for current Alzheimer's therapy $(29,30)$.

The role of the glucose-lowering effect of the antidiabetic medications is unclear. We do not see a reduced risk for all medication groups but only for some and we have no evidence that patients treated with for example the newer antidiabetic drugs were more well treated. In a subanalysis, we found no impact of glycaemic control (HbA1c). Also, hypoglycemia may mediate the relationship, but severe hypoglycemia remains rare in type 2 diabetes. In our analyses, we adjusted for number of hypoglycemic episodes registered at a hospital, and this did not change the results.

Our study has several strengths. We assessed use of antidiabetic medication from a nationwide Prescription Registry, which provided detailed long-term drug use histories and eliminated recall bias. The use of nationwide registries with nearly complete coverage and continuous updates on demographics, hospital contacts and dementia outcomes minimized selection bias and provided us with data to perform a nested case-control study including all diabetes patients diagnosed with dementia in Denmark. This design allowed us to handle the time-varying exposure without risk of immortal time bias (31). In our nested case-control study, we could calculate the exact amount of purchases of each drug for each patient which meant that we could also test whether a higher exposure was associated with dementia. Another strength of our study was that we were able to include register-based information on a number of relevant covariables including diabetes complications. The different antidiabetic medications were also mutually adjusted for limiting confounding by prescription choice. In a subgroup we could further adjust for clinical variables like HbA1c, low-density lipoprotein cholesterol, systolic blood pressure and so forth even though, unfortunately, they were not measured at the time of the diabetes diagnosis. However, we had no information on lifestyle such as physical activity, alcohol use and tobacco smoking or on social relations and, consequently, we cannot exclude that the odds could be 
explained by residual confounding from other factors. The validity of the dementia diagnoses in the Danish registers has previously been evaluated in two independent studies $(32,33)$. These studies found that for patients admitted in $2003,83 \%$ of the diagnosis were confirmed by an external assessor (32). The corresponding number was 70\% for patients admitted in 2008. The lowest validity was seen in the youngest population (33). Our study also has several potential limitations. First, our results could be biased if users of antidiabetic medication were more likely to get a dementia diagnosis, but this would implicate that our estimates would be even stronger. Further, the association could be explained by reverse causation: patients with an early stage of cognitive decline might less often be prescribed antidiabetic medications, especially the newer drugs (SGLT2, GLP1) might less often be prescribed to patients with early cognitive decline who have not yet received a diagnosis of dementia. However, such potential channeling bias does not seem to be present for glucoselowering drugs (34) and we found that patients with newer antidiabetic medications were relatively similar to those treated with older drugs. We also adjusted for a large number of comorbidities and clinical variables in order to eliminate any confounding by indication. Secondly, since the Prescription Registry was established in 1995 and does not include drugs received during hospitalization, antidiabetic medication use prior to 1995 or during hospital stay would be misclassified as non-use. Such assumed non-differential misclassification may have led to attenuation of risk estimates. Third, it is conceivable that, prior to diagnosis, dementia could influence adherence to medication use, for example, by affecting cognitive skills. However, any such bias would again lead to an underestimation of risks. Fourth, GLP1 analogs, DPP4 inhibitors, and SGLT2 inhibitors are all relatively new drugs and consequently follow-up time in these groups was shorter which could question causality but may indicate that these drugs postpone cognitive decline and dementia diagnosis.

In conclusion, we found that use of metformin, GLP1 analogs, DPP4 inhibitors, and SGLT2 inhibitors were associated with significantly lower odds of dementia in patients with type 2 diabetes. We propose to test the hypothesis that some especially newer antidiabetic medications may influence the risk of dementia related to diabetes in other populations and clinical trails.

\section{Supplementary data}

This is linked to the online version of the paper at https://doi.org/10.1530/ EJE-19-0259.

\section{Declaration of interest}

The authors declare that there is no conflict of interest that could be perceived as prejudicing the impartiality of this study.

\section{Funding}

The work was supported by Jaschafonden, the Lundbeck Foundation (Grant number R249-2017-1074), The Danish Medical Association (Grant number 22017-1064/26 HBN), the M.D. Gerhard Linds Grant, the Program for Clinical Research Infrastructure (PROCRIN) established by the Lundbeck Foundation and the Novo Nordisk Foundation, the Family Hede Nielsen Foundation, the Holger Rabitz and wife Doris Mary, born Phillip's Memorial Foundation, and the Danish Diabetes Association. The sponsors had no role in the design and conduct of the study; in the collection, management, analysis, and interpretation of the data; or in the preparation, review, or approval of the manuscript.

\section{Author contribution statement}

I W A made the first draft of the article. M W A was responsible for analyzing the data with help from I W A and M O. I W A is responsible for the funding and data collection of the study with help from M W A, M O, and M B J. All authors took part in design, interpretation of the results, and have read and approved the final version of the article. The corresponding author attests that all listed authors meet authorship criteria and that no others meeting the criteria have been omitted.

\section{Acknowledgements}

The authors thank Dr Roger S Mclntyre who has inspired us to make this study. Also, they want to thank Rikke Kart Jacobsen from PROCRIN, who helped establish our diabetes and reference population from the NDR.

\section{References}

1 Livingston G, Sommerlad A, Orgeta V, Costafreda SG, Huntley J, Ames D, Ballard C, Banerjee S, Burns A, Cohen-Mansfield J et al. Dementia prevention, intervention, and care. Lancet 2017390 2673-2734. (https://doi.org/10.1016/S0140-6736(17)31363-6)

2 Zhang J, Chen C, Hua S, Liao H, Wang M, Xiong Y \& Cao F. An updated meta-analysis of cohort studies: diabetes and risk of Alzheimer's disease. Diabetes Research and Clinical Practice 2017124 41-47. (https://doi.org/10.1016/j.diabres.2016.10.024)

3 Ninomiya T. Diabetes mellitus and dementia. Current Diabetes Reports 201414 487. (https://doi.org/10.1007/s11892-014-0487-z)

4 McClean PL \& Holscher C. Liraglutide can reverse memory impairment, synaptic loss and reduce plaque load in aged APP/PS1 mice, a model of Alzheimer's disease. Neuropharmacology 201476 57-67. (https://doi.org/10.1016/j.neuropharm.2013.08.005)

5 Hansen HH, Fabricius K, Barkholt P, Niehoff ML, Morley JE, Jelsing J, Pyke C, Knudsen LB, Farr SA \& Vrang N. The GLP-1 receptor agonist liraglutide improves memory function and increases hippocampal CA1 neuronal numbers in a senescence-accelerated mouse model of Alzheimer's disease. Journal of Alzheimer's Disease 201546 877-888. (https://doi.org/10.3233/JAD-143090)

6 Holscher C. The incretin hormones glucagonlike peptide 1 and glucose-dependent insulinotropic polypeptide are neuroprotective in mouse models of Alzheimer's disease. Alzheimer's and Dementia 201410 (1 Supplement) S47-S54. (https://doi.org/10.1016/j. jalz.2013.12.009)

7 Gejl M, Gjedde A, Egefjord L, Moller A, Hansen SB, Vang K, Rodell A, Braendgaard H, Gottrup H, Schacht A et al. In Alzheimer's disease, 6-month treatment with GLP-1 analog prevents decline of brain 
glucose metabolism: randomized, placebo-controlled, double-blind clinical trial. Frontiers in Aging Neuroscience 20168 108. (https://doi. org/10.3389/fnagi.2016.00108)

8 International Diabetes Federation. IDF Diabetes Atlas, 8th ed. Brussels, Belgium: International Diabetes Federation, 2017

9 Carstensen B, Kristensen JK, Marcussen MM \& BorchJohnsen K. The national diabetes register. Scandinavian Journal of Public Health 201139 (7 Supplement) 58-61. (https://doi. org/10.1177/1403494811404278)

10 Green A, Sortso C, Jensen PB \& Emneus M. Validation of the Danish national diabetes register. Clinical Epidemiology 20157 5-15. (https:// doi.org/10.2147/CLEP.S72768)

11 Schmidt M, Schmidt SA, Sandegaard JL, Ehrenstein V, Pedersen L, Sorensen HT. The Danish National Patient Registry: a review of content, data quality, and research potential. Clinical Epidemiology 20157 449-490. (https://doi.org/10.2147/CLEP.S91125)

12 WHO Collaborating Centre for Drug Statistics Methodology (WHOCC): DDD definition and general considerations. (available at: https://www.whocc.no/ddd/definition_and_general_ considera/)

13 Jorgensen ME, Kristensen JK, Reventlov Husted G, Cerqueira C, Rossing P. The Danish Adult Diabetes Registry. Clinical Epidemiology 20168 429-434. (https://doi.org/10.2147/CLEP.S99518)

14 Hsu CC, Wahlqvist ML, Lee MS \& Tsai HN. Incidence of dementia is increased in type 2 diabetes and reduced by the use of sulfonylureas and metformin. Journal of Alzheimer's Disease 201124 485-493. (https://doi.org/10.3233/JAD-2011-101524)

15 Cheng C, Lin CH, Tsai YW, Tsai CJ, Chou PH \& Lan TH. Type 2 diabetes and antidiabetic medications in relation to dementia diagnosis. Journals of Gerontology: Series A, Biological Sciences and Medical Sciences 201469 1299-1305. (https://doi.org/10.1093/ gerona/glu073)

16 Orkaby AR, Cho K, Cormack J, Gagnon DR \& Driver JA. Metformin vs sulfonylurea use and risk of dementia in US veterans aged $>/=65$ years with diabetes. Neurology 201789 1877-1885. (https://doi. org/10.1212/WNL.0000000000004586)

17 Markowicz-Piasecka M, Sikora J, Szydlowska A, Skupien A, MikiciukOlasik E \& Huttunen KM. Metformin - a future therapy for neurodegenerative diseases: theme: drug discovery, development and delivery in Alzheimer's disease Guest Editor: Davide Brambilla. Pharmaceutical Research 201734 2614-2627. (https://doi. org/10.1007/s11095-017-2199-y)

18 Umegaki H. Type 2 diabetes as a risk factor for cognitive impairment: current insights. Clinical Interventions in Aging 20149 1011-1019. (https://doi.org/10.2147/CIA.S48926)

19 Imfeld P, Bodmer M, Jick SS \& Meier CR. Metformin, other antidiabetic drugs, and risk of Alzheimer's disease: a populationbased case-control study. Journal of the American Geriatrics Society 2012 60 916-921. (https://doi.org/10.1111/j.1532-5415.2012.03916.x)

20 Cao B, Rosenblat JD, Brietzke E, Park C, Lee Y, Musial N, Pan Z, Mansur RB \& McIntyre RS. Comparative efficacy and acceptability of antidiabetic agents for Alzheimer's disease and mild cognitive impairment: a systematic review and network meta-analysis. Diabetes, Obesity and Metabolism 201820 2467-2471. (https://doi. org/10.1111/dom.13373)

21 Calsolaro V \& Edison P. Novel GLP-1 (glucagon-like Peptide-1) analogues and insulin in the treatment for Alzheimer's disease and other neurodegenerative diseases. CNS Drugs 201529 1023-1039. (https://doi.org/10.1007/s40263-015-0301-8)
22 Candeias EM, Sebastiao IC, Cardoso SM, Correia SC, Carvalho CI, Placido AI, Santos MS, Oliveira CR, Moreira PI \& Duarte AI. Gutbrain connection: the neuroprotective effects of the anti-diabetic drug liraglutide. World Journal of Diabetes 20156 807-827. (https:// doi.org/10.4239/wjd.v6.i6.807)

23 Gejl M, Brock B, Egefjord L, Vang K, Rungby J \& Gjedde A. Bloodbrain glucose transfer in Alzheimer's disease: effect of GLP-1 analog treatment. Scientific Reports 20177 17490. (https://doi.org/10.1038/ s41598-017-17718-y)

24 Nauck M. Incretin therapies: highlighting common features and differences in the modes of action of glucagon-like peptide- 1 receptor agonists and dipeptidyl peptidase- 4 inhibitors. Diabetes, Obesity and Metabolism 201618 203-216. (https://doi.org/10.1111/dom.12591)

25 Morales J. The pharmacologic basis for clinical differences among GLP-1 receptor agonists and DPP-4 inhibitors. Postgraduate Medicine 2011123 189-201. (https://doi.org/10.3810/pgm.2011.11.2508)

26 Nauck MA. Update on developments with SGLT2 inhibitors in the management of type 2 diabetes. Drug Design, Development and Therapy 20148 1335-1380. (https://doi.org/10.2147/DDDT.S50773)

27 Lin B, Koibuchi N, Hasegawa Y, Sueta D, Toyama K, Uekawa K, Ma M, Nakagawa T, Kusaka H \& Kim-Mitsuyama S. Glycemic control with empagliflozin, a novel selective SGLT2 inhibitor, ameliorates cardiovascular injury and cognitive dysfunction in obese and type 2 diabetic mice. Cardiovascular Diabetology 201413 148. (https://doi. org/10.1186/s12933-014-0148-1)

28 Sa-Nguanmoo P, Tanajak P, Kerdphoo S, Jaiwongkam T, Pratchayasakul W, Chattipakorn N \& Chattipakorn SC. SGLT2inhibitor and DPP-4 inhibitor improve brain function via attenuating mitochondrial dysfunction, insulin resistance, inflammation, and apoptosis in HFD-induced obese rats. Toxicology and Applied Pharmacology 2017333 43-50. (https://doi.org/10.1016/j. taap.2017.08.005)

29 Shaikh S, Rizvi SM, Shakil S, Riyaz S, Biswas D \& Jahan R. Forxiga (dapagliflozin): plausible role in the treatment of diabetes-associated neurological disorders. Biotechnology and Applied Biochemistry 201663 145-150. (https://doi.org/10.1002/bab.1319)

30 Rizvi SM, Shakil S, Biswas D, Shakil S, Shaikh S, Bagga P \& Kamal MA. Invokana (canagliflozin) as a dual inhibitor of acetylcholinesterase and sodium glucose co-transporter 2: advancement in Alzheimer's disease-diabetes type 2 linkage via an enzoinformatics study. CNS and Neurological Disorders Drug Targets 201413 447-451. (https://doi.org/ $10.2174 / 18715273113126660160)$

31 Etminan M. Pharmacoepidemiology II: the nested case-control study - a novel approach in pharmacoepidemiologic research. Pharmacotherapy 200424 1105-1109. (https://doi.org/10.1592/ phco.24.13.1105.38083)

32 Phung TK, Andersen BB, Hogh P, Kessing LV, Mortensen PB \& Waldemar G. Validity of dementia diagnoses in the Danish hospital registers. Dementia and Geriatric Cognitive Disorders 200724 220-228. (https://doi.org/10.1159/000107084)

33 Salem LC, Andersen BB, Nielsen TR, Stokholm J, Jorgensen MB, Rasmussen MH \& Waldemar G. Overdiagnosis of dementia in young patients - a nationwide register-based study. Dementia and Geriatric Cognitive Disorders 201234 292-299. (https://doi. org/10.1159/000345485)

34 Ankarfeldt MZ, Thorsted BL, Groenwold RH, Adalsteinsson E, Ali MS \& Klungel $\mathrm{OH}$. Assessment of channeling bias among initiators of glucose-lowering drugs: a UK cohort study. Clinical Epidemiology 2017 9 19-30. (https://doi.org/10.2147/CLEP.S124054)
Received 7 April 2019

Revised version received 8 July 2019

Accepted 18 July 2019 